\title{
Large Scale Membrane Movement Induced by a Cation Switch
}

\author{
Laura H. John, Gail. M. Preston, Mark S. P. Sansom and Luke A. Clifton*
}

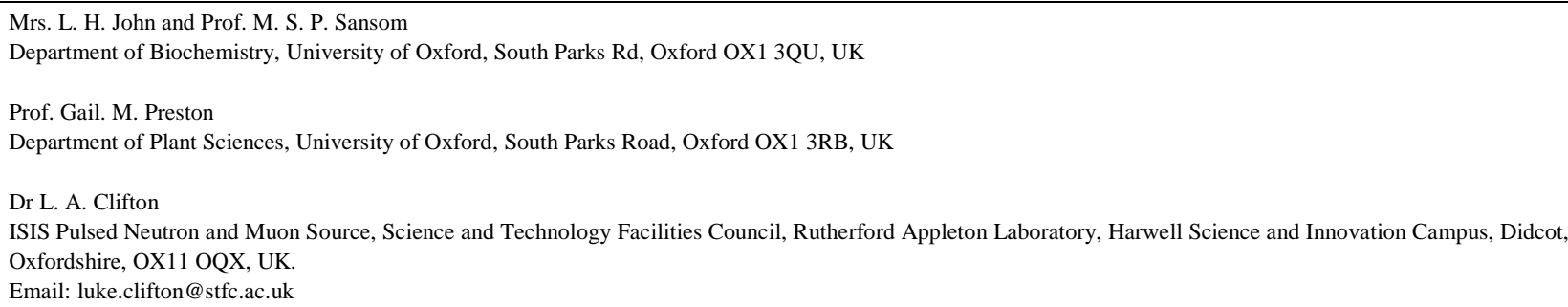

Abstract: A biomembrane sample system where millimolar changes of cations induce reversible large scale $(\geq 200 \AA)$ changes in the membrane-to-surface distance is described. The system composes of a freefloating bilayer (FFB), formed adjacent to a selfassembled monolayer (SAM). To examine the membrane movements, differently charged FFBs in the presence and absence of $\mathrm{Ca}^{2+}$ and $\mathrm{Na}^{+}$, respectively, were examined using neutron reflectivity (NR) and quartz crystal microbalance (QCM) measurements, alongside molecular dynamics (MD) simulations. In NR the variation of $\mathrm{Ca}^{2+}$ and $\mathrm{Na}^{+}$concentration enabled precision manipulation of the FFB-to-surface distance. Simulations suggest that $\mathrm{Ca}^{2+}$ ions bridge between SAM and bilayer whereas the more diffuse binding of $\mathrm{Na}^{+}$, especially to bilayers, is unable to fully overcome the repulsion between anionic FFB and anionic SAM. Reproduced NR results with QCM demonstrate the potential of this easily producible sample system to become a standard analysis tool for e.g. investigating membrane binding effects, endocytosis and cell signalling.

\section{Introduction}

Comprised predominantly of a lipid bilayer with embedded and bound proteins from a large variety of classes, biological membranes are the key structural material of life at the cellular level. Membranes define the external limit of each cell and, within eukaryotic cells, the many organelles, which carry out a multiplicity of cellular functions. Membranes control molecular transport, both into and out of the cell. Therefore, membrane associated proteins are involved in almost all biochemical pathways and account for around $\sim 40 \%$ of drug targets ${ }^{[1]}$. Studying membranes in vivo is challenging due to their complexity and small transverse size. Model membranes have therefore been key in gaining a molecular level understanding of membrane biochemistry ${ }^{[2],[3]}$. These systems allow for precision structural and biophysical studies to occur on systems of reduced (whilst defined) complexity compared to those found in vivo.

Planar supported lipid membrane samples at interfaces offer an ideal model membrane system due to the variety of benchtop techniques suitable for analysing this sample type ${ }^{[4]}$ and the relative ease of fabrication of these samples by vesicle rupture ${ }^{[5]}$, solvent exchange ${ }^{[6]}$ or monolayer transfer techniques ${ }^{[7]}$. The simplest models consist of bilayers deposited directly onto a 
solid support material such as silicon, glass or mica ${ }^{[8],[9]}$, More advanced forms of planar membrane models use soft polymer supports ${ }^{[10]}$ or surface tethers ${ }^{[1]]}$ to reduce surface influence and thus have water on both sides of the membrane model. Floating supported bilayers achieve this through a combination of repulsive and attractive forces between the membrane and the surface $^{[12],[13]}$. This effect causes the membrane to float $\sim 2 \mathrm{~nm}$ away from a bulk interface. Consequently a layer of 'bulk' solution is present on both sides of the planar membrane without the need for either tethering or interacting with surface bound material. Recently we discovered that membranes which float 1-3 nm away from a solid interface can be self-assembled via vesicle rupture adjacent to carboxylate terminated oligo (ethylene glycol) alkanthiol self-assembled monolayer surfaces (OEG-SAM) $)^{[14]}$. The self-assembly of the free floating bilayer (FFB) onto the support surface significantly reduces the complexity of the FFB generation protocol, thus opening this sample system up to wider utilisation. This is in contrast with previous systems which in general required LangmuirBlodgett/Langmuir Schaefer deposition to form high quality membranes, and therefore required specialist deposition apparatus and expertise, ${ }^{,[15],[16],[17],}$.

OEG-SAMs have gained significant interest due to their use in anti-fouling surface coatings ${ }^{[18]}$, with this effect related to the repulsive hydration forces resulting from water layers bound to the EG groups of the $\mathrm{SAM}^{[19],[20]}$. The most commonly studied OEG-SAM contains a terminal hydroxyl group and has, according to the "Whiteside" rules of hydrophilicity, no net charge ${ }^{[21],[22],[23]}$. Here, and in previous studies on FFB systems, we have used carboxyl terminated ( $\mathrm{COOH}-)$ OEG-SAMs as a support for FFBs. These do not obey these rules due to partial ionisation of the SAM terminal carboxyl groups $^{[24]}$. Charged surfaces are known to bind oppositely charged counter ions at the solid liquid interface, which plays a key role in many technological processes such as water purification ${ }^{[25]}$ and cation exchange chromatography ${ }^{[26]}$ However, the interactions of metal cations with partly negatively charged COOH-OEG-SAMs and its resulting surface charge modulation have not been studied.

In a previous study, we observed that the presence of $200 \mathrm{mM} \mathrm{NaCl}$ in the bulk solution reversibly increased the bilayer-to-SAM distance but could not identify the cause of this effect ${ }^{[14]}$. Here, we demonstrate a reversible phenomenon where removing and re-adding a calcium chloride $\left(\mathrm{CaCl}_{2}\right)$ concentration one hundred fold smaller than the $\mathrm{NaCl}$ concentration previously studied ( $2 \mathrm{mM}$ vs. $200 \mathrm{mM}$ ) induced substantial reversible changes to the bilayer-to-SAM distance on length scales of greater than $20 \mathrm{~nm}$. As electrolytes seem to have a major effect on the behaviour of the FFB system, and given that the self-assembly process of the FFB onto the $\mathrm{COOH}-\mathrm{OEG}-\mathrm{SAM}$ requires $\mathrm{CaCl}_{2}$ present in the buffer solution, the interactions of $\mathrm{Ca}^{2+}$ and $\mathrm{Na}^{+}$, as well as the water density within the system were of particular interest in this study. Therefore, $\mathrm{Ca}^{2+}$ or $\mathrm{Na}^{+}$ in the buffer solution and FFB lipid compositions with differing levels of biomemetic accuracy were used in the sample systems investigated.

Neutron reflectometry (NR) was used to examine the interfacial membrane constructs, due to its sensitivity as a structural probe for samples buried within complex environments, such as the solid-liquid flow cells used here. This technique is able to structurally resolve complex assemblies of macromolecules through the use of differential protium/deuterium labelling of the interfacial samples and bulk solution ${ }^{[15]}{ }^{[27]}$. To obtain a molecular level understanding of the membrane 
movements observed in the NR measurements, molecular dynamics (MD) simulations were undertaken to probe cation and water distribution profiles above the SAM and membranes, as well as cation binding energies to the COOH-OEG-SAM and the different lipid bilayer membranes used in the NR experiments. The combination of these two techniques allowed for a detailed understanding of the ion induced interfacial phenomena. Quartz crystal microbalance (QCM) measurements were additionally utilized to demonstrate the transfer of the bio-mimetic FFB sample system to studies using benchtop analytical techniques.

\section{Results and Discussion}

\section{NR Reveals Cation Induced Bilayer-to-SAM Distance \\ Modulation}

Self-assembled free floating membranes (FFBs) of different lipid compositions adjacent to $\mathrm{COOH}-\mathrm{OEG}$ SAMs were generated (Table 1). The complexity of the lipid composition was stepwise increased in order to investigate the feasibility of the system for studies of membrane systems of near-biological complexity. Therefore, starting from a POPC-membrane and a POPC/POPS mixture (8:2, mol:mol), a model of the inner leaflet of the mammalian plasma membrane (POPC/POPS/POPIP3 7:2:1, mol:mol) ${ }^{[28][29]}$ and a model approximating the plant plasma membrane (POPC/POPS/Chol/DGDG 5:2:2:1, mol:mol) ${ }^{[30][31]}$ were produced. As the self-assembling process of FFBs needed $2 \mathrm{mM} \mathrm{CaCl}_{2}$ present in the buffer solution and a previous study suggested a significant impact of cations on the bilayer-to-SAM distance, the role of the $\mathrm{Ca}^{2+}$ and its interactions within the system were of particular interest. Therefore, each system was treated with $1 \mathrm{mM}$ EDTA to remove $\mathrm{Ca}^{2+}$ after the membrane deposition. Then, to investigate the difference between divalent $\mathrm{Ca}^{2+}$ and monovalent $\mathrm{Na}^{+}, 200 \mathrm{mM} \mathrm{NaCl}$ was added to the system. Finally, the system was again flushed with a $2 \mathrm{mM} \mathrm{CaCl}_{2}$ containing solution. NR measurements at three isotopic contrasts $\left(\mathrm{H}_{2} \mathrm{O}, \mathrm{AuMW}\right.$ and $\left.\mathrm{D}_{2} \mathrm{O}\right)$ were carried out for each system directly after the FFB deposition and after each single treatment. The data fitting quality was high for all the samples studied, which allowed us to resolve the relative distributions of the COOH-OEG-SAM, the membrane, and the water components under all conditions.

An example of a full NR data set, including the model data fits and the neutron scattering length density (nSLD) profiles, are shown for the mammalian plasma model membrane (POPC/POPS/DOPIP3 7:2:1, mol:mol) in Figure 1. For all FFB samples the bilayerto-SAM distance, together with the FFB roughness, are listed in Table 1. Here, the bilayer-to-SAM distance is defined as that between the COOH-OEG-SAM/water interface and the adjacent bilayer head-group/water interface. Therefore, the bilayer-to-SAM distance is equivalent to the thickness of the water interlayer between the FFB and the COOH-OEG-SAM surface. Further key structural parameters of the NR experiments are listed in Table S2. All FFBs showed high bilayer coverage and were in good agreement with previous studies ${ }^{[14],[32] \text {. }}$ 

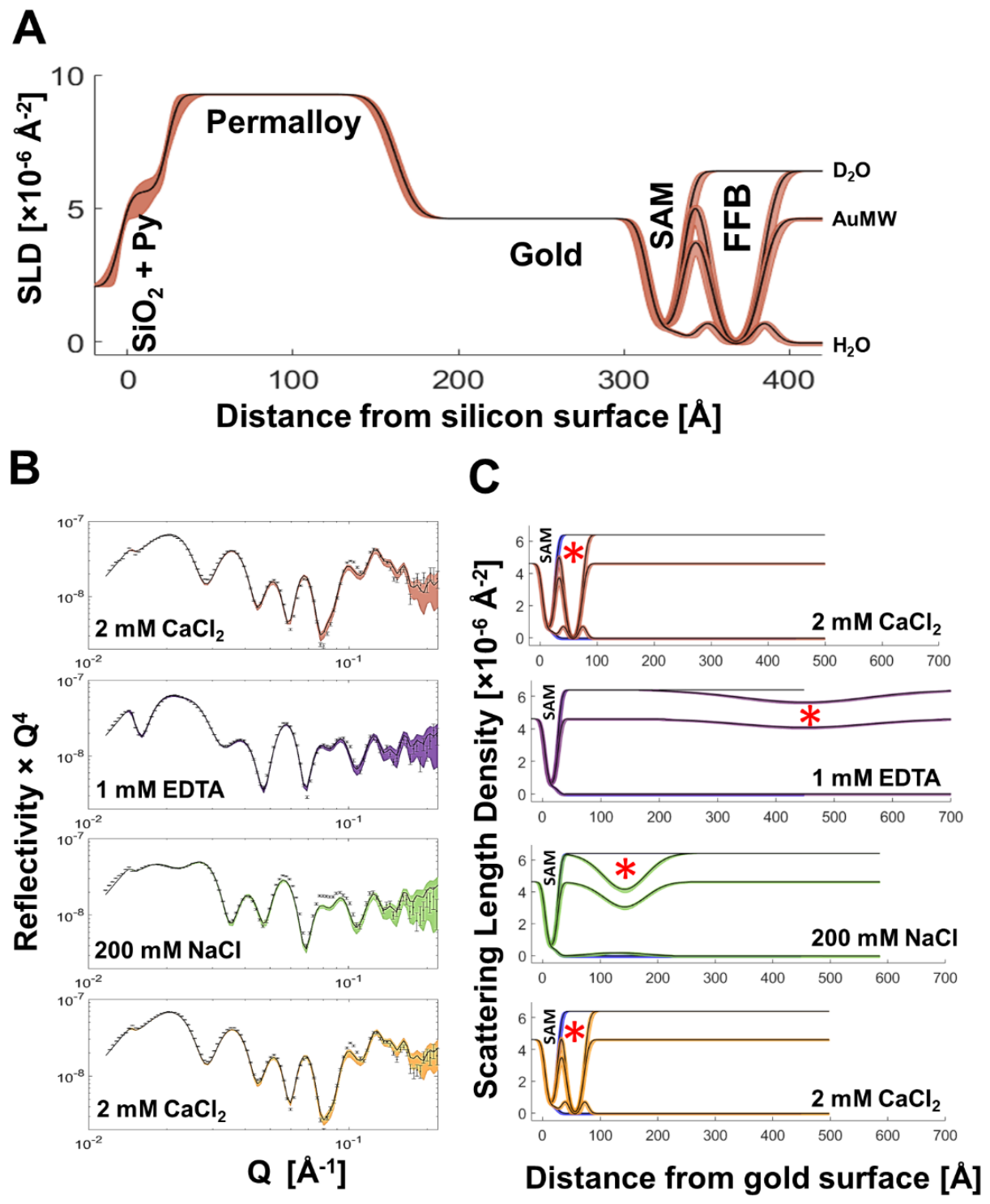

Figure 1 | SLD and Reflectivity Profiles of a Mammalian Plasma Model Membrane in Absence and Presence of $\mathrm{Ca}^{2+}$ and $\mathrm{Na}^{+}$, respectively: (A) Full range scattering length density (SLD) profiles of the POPC:POPS:DOPIP3 (7:2:1, mol:mol) free floating bilayer (FFB) adjacent to a COOH-OEG-SAM in the presence of $2 \mathrm{mM} \mathrm{CaCl}_{2}$ are shown for the $\mathrm{D}_{2} \mathrm{O}, \mathrm{H}_{2} \mathrm{O}$ and AuMW isotopic contrast. Zero on the $\mathrm{x}$-axis is equal to the silicon/permalloy (Py) interface. (B, C) Reflectivity data of the POPC:POPS:DOPIP3 (7:2:1, mol:mol) FFB, including fits are shown for the $\mathrm{D}_{2} \mathrm{O}$ isotopic contrast in $\mathbf{B}$. The corresponding SLD profiles are shown in C. For clarity, the substrate layers of silicon, permalloy and gold are excluded and zero on the $\mathrm{x}$ axis is equal to the gold/COOH-OEG-SAM interface. Different colours represent different buffer solutions, containing: $2 \mathrm{mM}$ $\mathrm{CaCl}_{2}$ (red), $1 \mathrm{mM}$ EDTA (purple), $200 \mathrm{mM} \mathrm{NaCl}$ (green) and again added $2 \mathrm{mM} \mathrm{CaCl}_{2}$ (orange). The changing position of the bilayer under differing solution salt conditions is shown by a red asterisk $(*)$.

All FFB samples showed the same dependency of the bilayer-to-SAM distance and the FFB roughness on the cations present in the buffer solution (see Figure 2). The initial bilayer-to-SAM distance in the presence of $2 \mathrm{mM}$
$\mathrm{CaCl}_{2}$ was at around 10 to $15 \AA$ A. EDTA-mediated sequestration of $\mathrm{Ca}^{2+}$ resulted in a large increase of the bilayer-to-SAM distance to above $200 \AA$. The addition of $200 \mathrm{mM} \mathrm{NaCl}$ reduced the bilayer-to-SAM distance 
to an intermediate value, whilst returning the FFB into a $2 \mathrm{mM} \mathrm{CaCl}_{2}$ containing buffer solution returned the FFB back to its initial position adjacent to the membrane ( 10-15 $)$. In all cases the reversible movement of the FFB away from and back to the COOH-OEG-SAM surface did not result in measurable loss of the total membrane coverage, suggesting these to be fully reversible processes (see Table S2).

Though the trends in bilayer-to-SAM distance change against solution salt conditions were similar, the magnitude differed between FFBs. For example, whilst after $\mathrm{Ca}^{2+}$ sequestration the POPC bilayer moved to 200 $\AA$ from the COOH-OEG-SAM surface (see Table 1), the anionic eukaryotic membrane models (i.e. POPC:POPS and POPC:POPS:DOPIP3 ) were found to move to significantly higher distances relative to the COOH-OEG-SAM in this condition ( 600 and $\sim 400 \AA$ respectively, see Table 1). This suggested a correlation between membrane anionic character and the bilayerto-SAM distance change after $\mathrm{Ca}^{2+}$ sequestration. A similar trend was seen with $200 \mathrm{mM} \mathrm{NaCl}$. Here, the POPC sample returned to a close membrane-to-SAM surface distance $(\sim 35 \AA$, compared to $\sim 10 \AA$ in $2 \mathrm{mM}$ $\mathrm{CaCl}_{2}$ ) whereas the anionic POPC:POPS and POPC:POPS:DOPIP3 membranes returned to a larger bilayer-to-SAM distance of $\sim 100 \AA$. Interestingly, when comparing the two mammalian anionic membrane models with each other, the bilayer-to-SAM distances during all applied salt solution buffer conditions were greater for the POPC:POPS membrane than for the
POPC:POPS:DOPIP3 membrane, although the latter had a higher net negative charge (see Table 1).

The anionic plant plasma membrane model (POPC:POPS:Chol:DGDG, 5:2:2:1, mol:mol) showed the same behaviour as the mammalian plasma membrane models, starting with a close position to the COOH-OEG-SAM in the presence of $\mathrm{Ca}^{2+}(15.0 \AA$ (12.9 $\AA, 17.4 \AA)$ ), followed by dramatic changes in the reflectivity profile upon EDTA mediated $\mathrm{Ca}^{2+}$ sequestration (see data in Figure SI 6). However, the interfacial structure could not be adequately resolved for this cation-free state, most likely due to the roughness of the membrane being too high for analysis or high heterogeneity in the membrane position at this solution condition. Nevertheless, the bilayer-to-SAM distance could be resolved once the sample was immersed into $200 \mathrm{mM} \mathrm{NaCl}$, showing a distance of 120 A (see Table 1).

In all cases, the bilayer roughness, which describes the height-height correlation function across a layer boundary ${ }^{[14]}$, showed a proportional increase with bilayer-to-SAM distance (see Table 1). In a continuous planar material such as a supported lipid bilayer the largest and therefore overriding source of roughness is likely to be displacement of the bilayer from its mean averaged position due to the fluctuations in the plane of the membrane ${ }^{[33]}$. This increase in the roughness value is therefore likely to be related to an increased in the fluctuation amplitude of the floating membrane as it moves away from the bulk interface ${ }^{[8],[13],}$. 


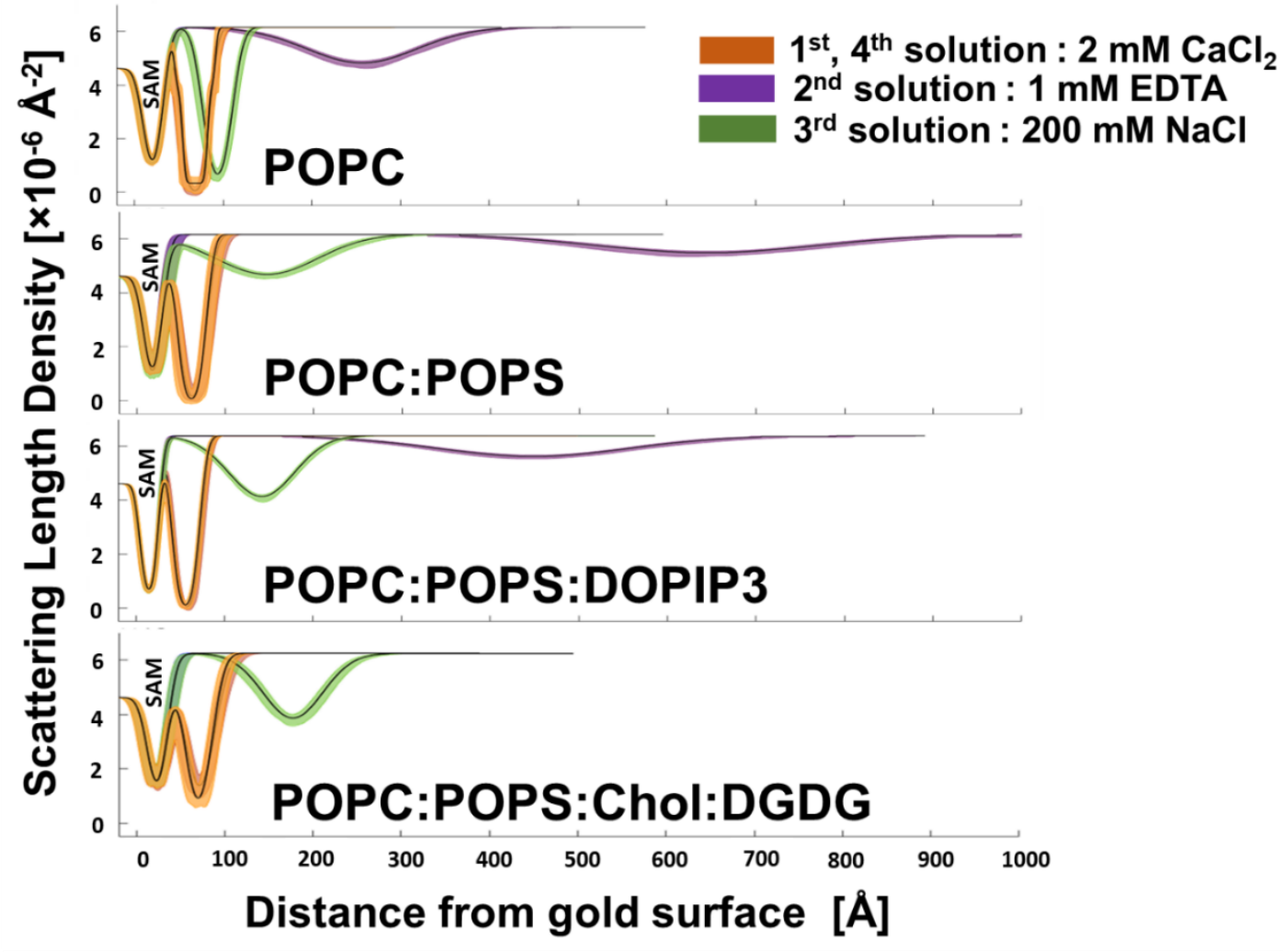

Figure 2 | SLD Profiles of Self-Assembled Free Floating Bilayers (FFBs) at Different Solution Salt Conditions: Each subplot shows the SLD profiles belonging to one respective membrane (see labels in subplots). Different colours of the profiles represent different additions to the buffer solution, which were present in each sample in following order: $2 \mathrm{mM} \mathrm{CaCl} 2$ after self-assembling process of the FFB (orange), no cations due to 1 mM EDTA mediated $\mathrm{Ca}^{2+}$ sequestration (purple), $200 \mathrm{mM} \mathrm{NaCl}$ (green) and finally, $2 \mathrm{mM} \mathrm{CaCl}_{2}$ again (orange). Changes in the bilayer-to-SAM distance can be seen through changes in the Gaussian distribution of the FFB (here equal to unlabelled distribution in SLDs). The largest changes caused the EDTA mediated $\mathrm{Ca}^{2+}$ sequestration, resulting in very rough FFBs, far away from the COOH-OEG-SAM surface. $200 \mathrm{mM} \mathrm{NaCl}$ returned the POPC bilayer almost completely, the anionic membranes only partly back towards their initial positions. In presence of negatively charged phospholipids the changes in bilayer-to-SAM distance were larger after $\mathrm{Ca}^{2+}$ sequestration (purple) and $\mathrm{Na}^{+}$addition (green), but similar after $\mathrm{Ca}^{2+}$ addition (SLD profiles overlaid in orange). Experimental data and model data fits used to produce these SLD profiles are given in supporting information Figures S3 to S6. 
Table 1 | Resolved Key NR Structural Parameters for Studied FFB Samples: The bilayer-to-SAM distance and the bilayer roughness of the different free floating bilayers (FFBs) are given for each applied solution salt condition. Parameter ranges as 95\% confidence intervals determined from MCMC resampling of the experimental data fits are given in brackets.

\begin{tabular}{|c|c|c|c|c|c|c|c|c|}
\hline \multirow[b]{2}{*}{ Bilayer } & \multicolumn{8}{|c|}{ Conditional Bilayer-to-SAM Distance and Bilayer Roughness } \\
\hline & $\begin{array}{c}\text { Distance } \\
2 \text { mM CaCl} \\
\text { (initial) } \\
{[\AA]}\end{array}$ & $\begin{array}{c}\text { Roughness } \\
2 \text { mM } \\
\mathbf{C a C l}_{2} \\
\text { (initial) } \\
{[\AA]}\end{array}$ & $\begin{array}{c}\text { Distance } \\
\text { EDTA } \\
\text { [A] }\end{array}$ & $\begin{array}{c}\text { Roughness } \\
\text { EDTA } \\
{[\AA]}\end{array}$ & $\begin{array}{c}\text { Distance } \\
200 \mathrm{mM} \mathbf{~ N a C l} \\
[\AA]]\end{array}$ & $\begin{array}{c}\text { Roughness } \\
200 \mathrm{mM} \\
\mathrm{NaCl} \\
{[\AA]}\end{array}$ & $\begin{array}{c}\text { Distance } \\
2 \mathrm{mM} \mathrm{CaCl} \\
\text { (final) } \\
{[\AA]}\end{array}$ & $\begin{array}{c}\text { Roughness } \\
\mathbf{2} \text { mM } \\
\mathbf{C a C l}_{2} \\
\text { (final) } \\
\text { [̊̊] }\end{array}$ \\
\hline POPC & $\begin{array}{c}12.7 \\
(11.0,15.8)\end{array}$ & $\begin{array}{c}5.4 \\
(3.9,6.7)\end{array}$ & $\begin{array}{c}200.9 \\
(194.7,206.9)\end{array}$ & $\begin{array}{c}62.5 \\
(60.0,65.3)\end{array}$ & $\begin{array}{c}38.2 \\
(36.4,40.3)\end{array}$ & $\begin{array}{c}8.5 \\
(7.2,9.8)\end{array}$ & $\begin{array}{c}12.8 \\
(11.1,15.1)\end{array}$ & $\begin{array}{c}2.5 \\
(0.2,5.2)\end{array}$ \\
\hline $\begin{array}{c}\text { POPC:POPS } \\
8: 2\end{array}$ & $\begin{array}{c}11.6 \\
(9.6,13.4)\end{array}$ & $\begin{array}{c}7.5 \\
(6.5,8.5)\end{array}$ & $\begin{array}{c}593.63 \\
(575.6,611.3)\end{array}$ & $\begin{array}{c}124.6 \\
(113.0,137.5)\end{array}$ & $\begin{array}{c}100.1 \\
(93.2 \AA, 106.8)\end{array}$ & $\begin{array}{c}54.7 \\
(51.2,59.1)\end{array}$ & $\begin{array}{c}10.8 \\
(8.5,12.7)\end{array}$ & $\begin{array}{c}6.0 \\
(4.3,7.5)\end{array}$ \\
\hline $\begin{array}{c}\text { POPC:POPS:DOPIP3 } \\
\text { 7:2:1 } \\
\text { (mammalian) }\end{array}$ & $\begin{array}{c}11.4 \\
(10.0,12.5)\end{array}$ & $\begin{array}{c}6.1 \\
(5.2,6.9)\end{array}$ & $\begin{array}{c}402.2 \\
(391.5,413.5)\end{array}$ & $\begin{array}{c}109.3 \\
(102.2,117.0)\end{array}$ & $\begin{array}{c}96.9 \\
(94.1,99.7)\end{array}$ & $\begin{array}{c}34.9 \\
(33.1,36.8)\end{array}$ & $\begin{array}{c}9.6 \\
(8.2,10.7)\end{array}$ & $\begin{array}{c}6.1 \\
(4.9,7.0)\end{array}$ \\
\hline $\begin{array}{c}\text { POPC:POPS:Chol:DGDG } \\
\text { 5:2:2:1 } \\
\text { (plant) }\end{array}$ & $\begin{array}{c}15.0 \\
(12.9,17.4)\end{array}$ & $\begin{array}{c}12.3 \\
(10.7,13.9)\end{array}$ & $\begin{array}{l}\text { Could not be } \\
\text { determined }\end{array}$ & $\begin{array}{c}\text { Could not be } \\
\text { determined }\end{array}$ & $\begin{array}{c}121.3 \\
(118.0,124.5)\end{array}$ & $\begin{array}{c}31.3 \\
(29.6,34.2)\end{array}$ & $\begin{array}{c}13.6 \\
(11.5,16.1)\end{array}$ & $\begin{array}{c}9.7 \\
(8.3,11.1)\end{array}$ \\
\hline
\end{tabular}


Finally, as the NR data suggested that $\mathrm{Ca}^{2+}$ brings the FFB much closer to the COOH-OEG-SAM surface than $\mathrm{Na}^{+}$, the effect of $\mathrm{Ca}^{2+}$ on the bilayer-to-SAM distance in the presence of physiological concentrations of $\mathrm{Na}^{+}$ was investigated. To this end, a POPC FFB was sequentially examined in buffer solutions containing $100 \mathrm{mM} \mathrm{NaCl}$ with and without $1 \mathrm{mM} \mathrm{CaCl}_{2}$. The solution without $\mathrm{CaCl}_{2}$ contained $0.5 \mathrm{mM}$ EDTA to remove any contaminating $\mathrm{Ca}^{2+}$ cations from the previous buffer solution. The NR analysis (see Figure 3) revealed that the bilayer-to-SAM distance was significantly smaller with the buffer solution containing both $\mathrm{Ca}^{2+}$ and $\mathrm{Na}^{+}$than in the presence of $\mathrm{Na}^{+}$only $(\sim 23$ $\AA$ vs. $\sim 41 \AA$ ). These data demonstrate the strong influence of divalent cations on the bilayer-to-SAM distance, even in the presence of a one hundred times higher concentration of monovalent cations. Furthermore, these results suggested the possibility of fine-tuning the bilayer-to-SAM distance by using combinations of mono- and divalent cations. (Figure S7 shows an independent repeat of this experiment, confirming the reproducibility of these effects).
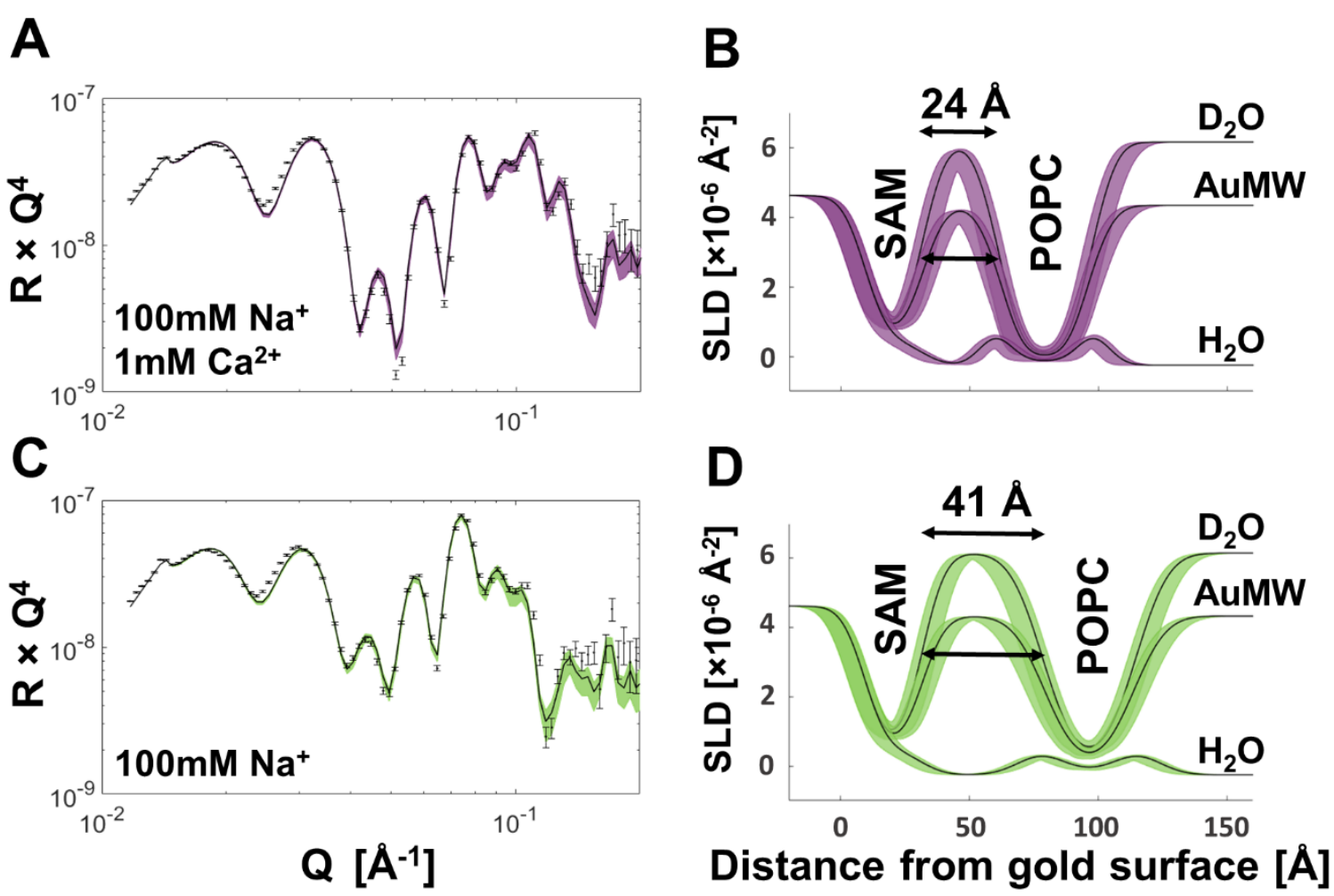

Figure $3 \mid \mathrm{Ca}^{2+}$ Dependent Fine-tuning of Bilayer-to-SAM Distance in Presence of $\mathrm{Na}^{+}$: (A and $\mathbf{C}$ Reflectivity profiles including model data fits, as well as the SLD profiles these fits describe (B and $\mathbf{D})$ are shown for the $\mathrm{D}_{2} \mathrm{O}$ contrast of a POPC free floating bilayer at two different solution salt conditions: $100 \mathrm{mM} \mathrm{NaCl} / 1 \mathrm{mM} \mathrm{CaCl}_{2}$ (A and $\mathbf{B}$, purple) and $100 \mathrm{mM}$ $\mathrm{NaCl} / 0.5 \mathrm{mM}$ EDTA ( $\mathbf{C}$ and $\mathbf{D}$, green). The resolved bilayer-to-SAM distances are given in ( $\mathbf{B}$ and $\mathbf{D}$ ), highlighting the difference in this caused by $1 \mathrm{mM} \mathrm{CaCl}_{2}$ in the presence of $100 \mathrm{mM} \mathrm{NaCl}$. A repeat of these measurements is given in Figure S7 and the relevant parameters in Table S4.

\section{Complementary Analysis on QCM-D Sensor Surfaces}

QCM-D is a surface sensitive technique utilising the piezoelectric effect for real-time measurements of surface interactions. The change in the resonant frequency $(\Delta f)$ is inversely proportional to the amount of mass at the sensor surface, as given by the Sauerbrey 
equation $^{[34],[35]}$. To complement NR measurements and to demonstrate utilisation of this sample system in studies using benchtop analytical techniques, FFBs of POPC:POPS (8:2, mol:mol) were self-assembled onto an COOH-OEG-SAM coated QCM sensor chip surface. Measurements of changes in the frequency $(\Delta f)$ and dissipation $(\Delta d)$ were used to monitor the sample response after changing the solution salt condition. This is based on the observation that the solution between the FFB and the COOH-OEG-SAM surface is trapped and therefore part of the total layer mass ${ }^{[5]}$. Therefore, the changes in bilayer-to-SAM distance will be associated with a decrease in $\Delta \mathrm{f}$ and an increase in $\Delta d$. This assumption is supported by the observation that the water inside a vesicle adsorbing onto a QCM sensor adds to the adsorbed mass measured and its loss can be measured during vesicle rupture ${ }^{[36]}$.

The QCM-D results were complementary to those observed with NR, showing a large decrease in $\Delta f$ and increase in $\Delta d$ when the buffer condition was changed from $2 \mathrm{mM} \mathrm{CaCl}_{2}$ to $0 \mathrm{mM} \mathrm{CaCl} 2$ by EDTA mediated $\mathrm{Ca}^{2+}$ sequestration. This implies that a thicker water layer was trapped between the COOH-OEG-SAM and the FFB in case of the $\mathrm{Ca}^{2+}$ free state compared to the initial state given in the presence of $\mathrm{Ca}^{2+}$ (see Figure 4). Interestingly, $\Delta f$ often after an initial decrease showed a slow increase, whereas $\Delta d$ increased always to a plateau after $\mathrm{Ca}^{2+}$ sequestration. It is possible that this effect was due to the movement of the FFB to a large distance, which was outside the frequency depth sensitivity range of QCM-D (which is inversely proportional to square of the resonant frequency) and/or at which the water was no longer mechanically coupled to the FFB. Besides the distance, increased membrane curvature and fluctuation amplitude of the FFB could have been another cause of the reduction in mechanically coupled water to the FFB. Immersing the FFB into $200 \mathrm{mM} \mathrm{NaCl}$ containing buffer solution seemed to bring the FFB partly back to the COOH-OEG-SAM, revealed by values of $\Delta f$ and $\Delta d$ between that observed in the presence of $\mathrm{Ca}^{2+}$ and the cation-free state. Finally, when the samples were exchanged back into $2 \mathrm{mM} \mathrm{CaCl}_{2}$ containing buffer solution the $\Delta f$ and $\Delta d$ values returned to their initial values. This suggested, like the NR data indicated, a reversible process of FFB-movement away from and back to a close position to the SAM. The solution exchange process was repeated twice for each sample and showed good reversibility, as well as robustness of the biomimetic sample system to repeated large distance changes (see Figure S9 for independent repeat and Figure S8 for first cycle of these measurements on the same sample systems demonstrating reproducibility between samples as well as within samples). 


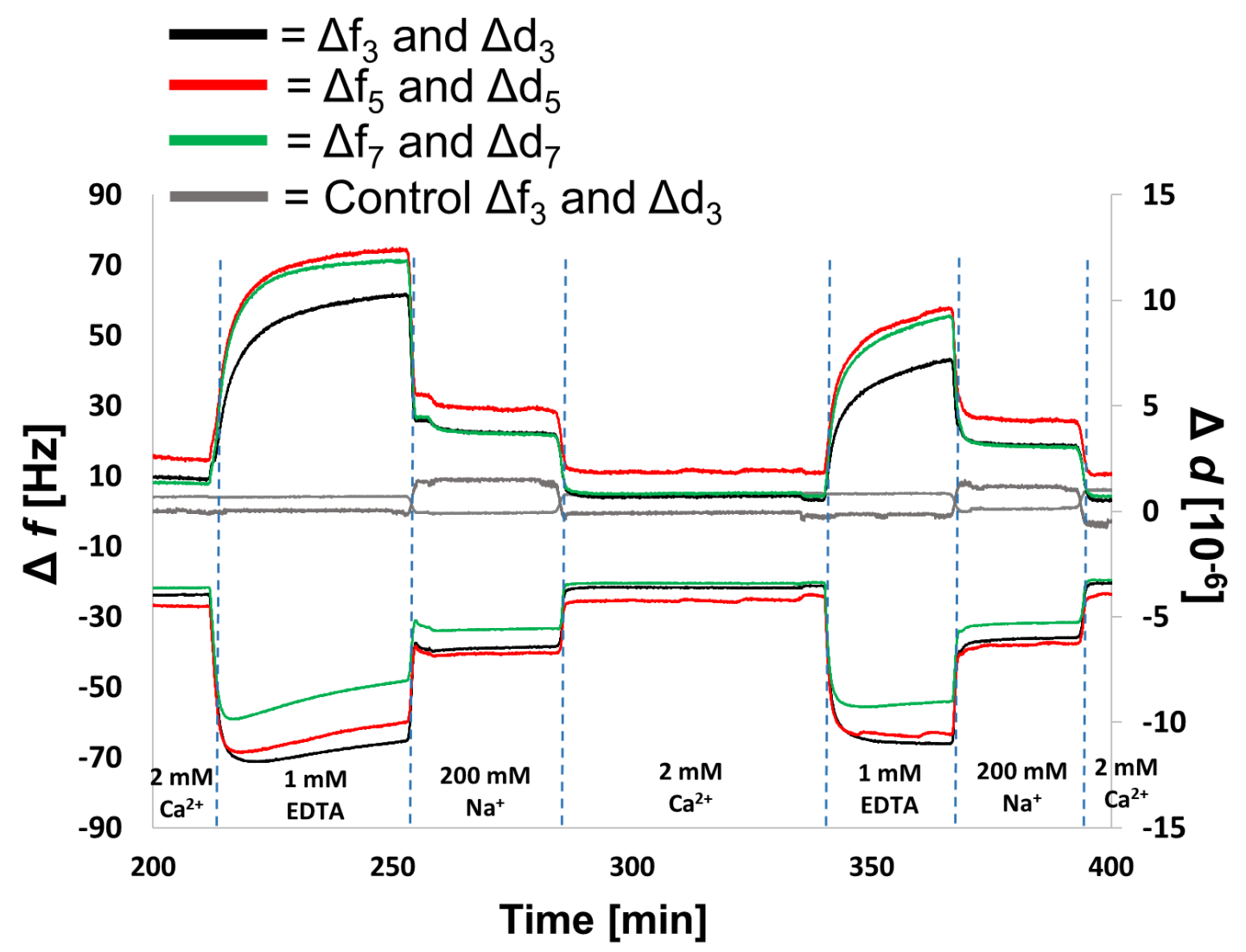

Figure 4 | QCM-D Measurements of a Free Floating POPC: POPS Bilayer (8:2, mol:mol) in absence and presence of $\mathrm{CaCl}_{2}$ and $\mathrm{NaCl}$ respectively: The changes in frequency $(\Delta f)$ and dissipation $(\Delta d)$ at different solution salt conditions are given for the $3^{\text {rd }}$ (black), $5^{\text {th }}$ (red) and $7^{\text {th }}$ (green) overtones. For the control data set with no membrane adjacent to the $\mathrm{COOH}-$ OEG-SAM only the $3^{\text {rd }}$ overtone is given (grey line). Repeated data sets are given in Figure S8-9.

MD Simulations Reveal Differences in Ion Binding between COOH-OEG-SAM and Membranes

Taken together, the NR and QCM-D results indicated that $\mathrm{Ca}^{2+}$ reversibly moves the FFB closest to the COOH-OEG-SAM, followed by $\mathrm{Na}^{+}$. These results raised the question what the difference is between $\mathrm{Na}^{+}$and $\mathrm{Ca}^{2+}$-interactions within the system. In particular, we wished to understand which layer preferentially interacted with cations, and how strongly the different cationic species interacted. This information would enable us to understand the observed substantive, reversible movements of the FFB away from the surface in response to changes in cation concentrations.
MD simulations of the COOH-OEG-SAM and of the different lipid bilayers used in the NR experiments were performed, at different $\mathrm{CaCl}_{2}$ and $\mathrm{NaCl}$ concentrations. Coarse grained (CG) simulations were used to generate initial model structures, using the well established Martini force field ${ }^{[37],[38]}$. For the CG model of the COOH-OEG-SAM a range of initial lattice constants $\left(a_{0}\right)$ of the hexagonal COOH-OEG-SAM-grid $(0.1 \AA$ intervals between 4.9 and $5.6 \AA$ ) were defined for a set of COOH-OEG-SAMs differing in the percentage of charged SAM-COO ${ }^{-}$molecules present $(0,5,10,20,39$ and $50 \%$ ). Figure 5 shows a schematic of a partly negatively charged COOH-OEG-SAM. Three replicas each of $60 \mathrm{~ns}$ production time for 48 different initial 
configurations were carried out. The lattice constant $\langle a\rangle$, tilt angle $\langle\theta\rangle$ of the alkane thiol chain (estimated via order parameters between the thiol-bead and the upper $\mathrm{CH}$-chain-bead $\left.\left\langle S_{T H I-C l B}\right\rangle\right)$, the monolayer thickness $\langle d\rangle$ and the surface hydrophilicity $\langle\eta\rangle$ were monitored to characterize the resultant $\mathrm{COOH}-\mathrm{OEG}-$
SAM compared to experiment. The averaged results of the last $50 \mathrm{~ns}$ of the $\mathrm{CG}$ production simulations are shown in Figure S11, with the errors representing the standard deviation between the three independent simulations of each system.

A
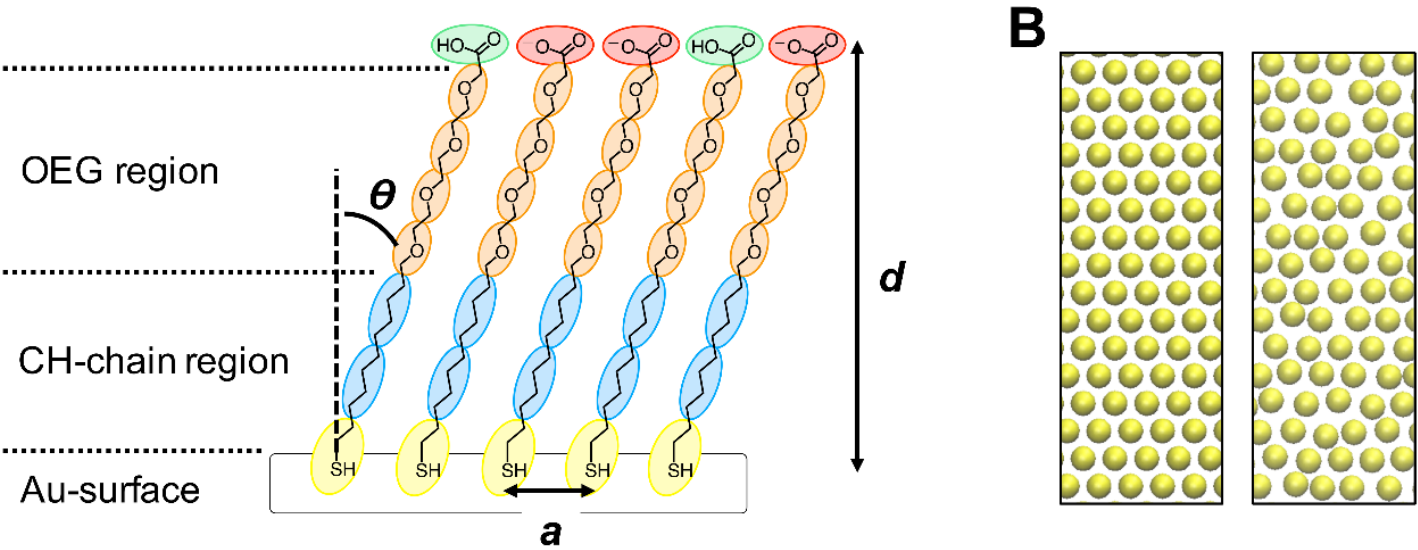

C

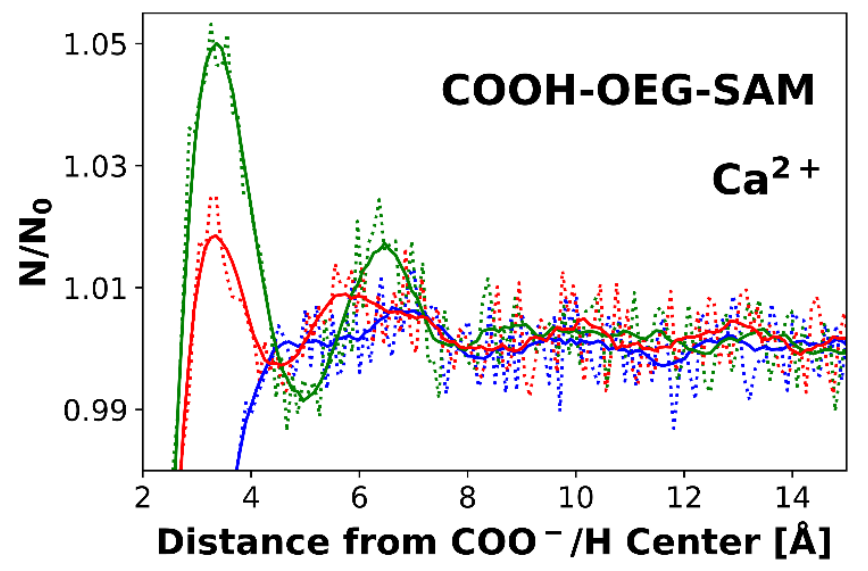

D

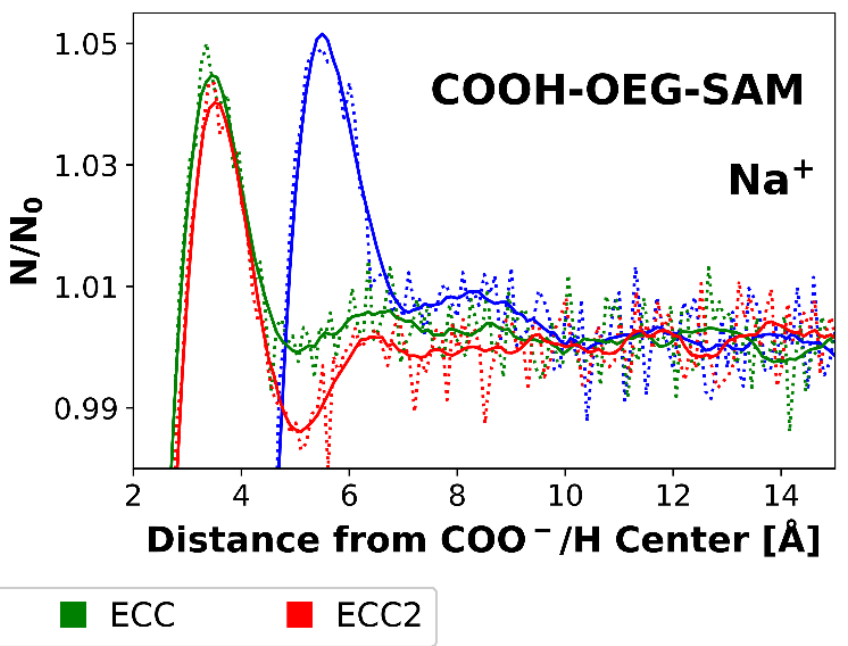

Figure 5 | Structure of COOH-OEG-SAM and Water Distribution Functions Adjacent to an All Atom (AA) Model of COOH-OEG-SAM: (A) Schematic structure of COOH-OEG-SAM, assembled onto the gold-sputtered surface, including the chemical structure and CG mapping schemes of the SAM molecules. Colours represent different Martini beads. Yellow: SC5, blue: C1, orange: SN0, red: SQn, green: SP2. Calculated parameters like thickness (d), lattice constant (a) and tilt angle ( $\theta)$ of the $\mathrm{COOH}-\mathrm{OEG}-\mathrm{SAM}$ are indicated. (B) Bottom view of the initial positioning of the SC5-beads of the SAM-molecules along a hexagonal grid (left), as well as after a $60 \mathrm{~ns}$ CG production simulation (right). (C, D) $N / N_{0}$ water molecules along surface normal with $N$ being the number of molecules at given z-position and $N_{0}$ the bulk concentration as average of number density above $4 \mathrm{~nm}$ away from the surface. (C) Results for samples with $400 \mathrm{mM} \mathrm{Ca}^{2+}$ and (D) with $400 \mathrm{mM} \mathrm{Na}^{+}$. Different colours represent three applied Charmm36 force field parameters (see Methods for details): Standard (blue), ECC (green), ECC2 (red). Zero on the $\mathrm{x}$-axis is equal to the centre of mass of the SAM carboxyl groups. 
Charged $\mathrm{SAM}_{-} \mathrm{COO}^{-}$and uncharged SAM-COOH molecules showed negligible differences in order parameters $\left\langle S_{T H I-C l B}\right\rangle$ within all SAM compositions (Figure S11, A/B). This explains why $\langle a\rangle$ and $\langle d\rangle$ seemed to be independent of the percentage of charged COOH-OEG-SAM molecules (Figure S11, C/D). Thus, for further simulations the $39 \%$ charged SAM was chosen, corresponding to the COOH-OEG-SAM used in $\mathrm{NR}$ at $7.2 \mathrm{pH}$ employing the pKa value of carboxyl terminated SAMs predicted by previous studies. ${ }^{[24]}$ However, $\langle d\rangle$ and $\left\langle S_{T H I-C l B}\right\rangle$ showed a high dependency on $a_{0}$. Previous studies showed that with the Martini bead definition it is not possible to reproduce the experimental alkane thiol chain tilt angle of 20-35 ${ }^{[39],[40]}$ while matching the experimental lattice constant of $4.97 \AA^{[41]}$ on gold (111). ${ }^{[42]}$ This was resolved when converting the $\mathrm{CG}$ presentation into an all-atom (AA) representation by rescaling the $x y$ coordinates by $0.93 x$. A tilt angle of $20-35^{\circ}$ corresponds to $0.51-0.82\left\langle S_{\text {THI-ClB }}\right\rangle$ which needed $a_{0}$ to be bigger than $5.1 \AA$ (Figure S11, A/B). An initial lattice constant of 5.2-5.3 $\AA$ agreed well with the COOH-OEG-SAM thickness observed in NR (20.3-21.7 $\AA$, Table S5 and Figure S11, D). The averaged $\langle a\rangle$ showed the overall structural stability of the packing (Figure S11, C) and revealed that both $a_{0}$ of 5.2 and $5.3 \AA$ converged due to minor rearrangements to $5.3 \AA$. Thus, an $a_{0}$ of $5.3 \AA$ was chosen for further simulations. The hydrophilicity of the SAM seemed to be almost independent of $a_{0}$ (Figure $\mathrm{S} 11, \mathrm{E})$.

The CG COOH-OEG-SAM was converted into an AA representation and simulated for up to $100 \mathrm{~ns}$ in the presence of different $\mathrm{Na}^{+}$and $\mathrm{Ca}^{2+}$ concentrations. To explore the robustness of our results to the parametrization of these ions we explored a number of variations, namely: Charmm $36^{[43]}$, the electronic continuum correction (ECC) within Charmm $36^{[44]}$, and a modified ECC version developed using Charmm36, here called $\mathrm{ECC}^{[45]}$ (see section S1.7 for details). As anticipated, we observed oscillations in the water density close to the COOH-OEG-SAM surface, extending approximately four molecular water diameters away from the surface (Figure 5, C/D), in agreement with previous studies on OEG-SAMs ${ }^{[46],[47]}$. 

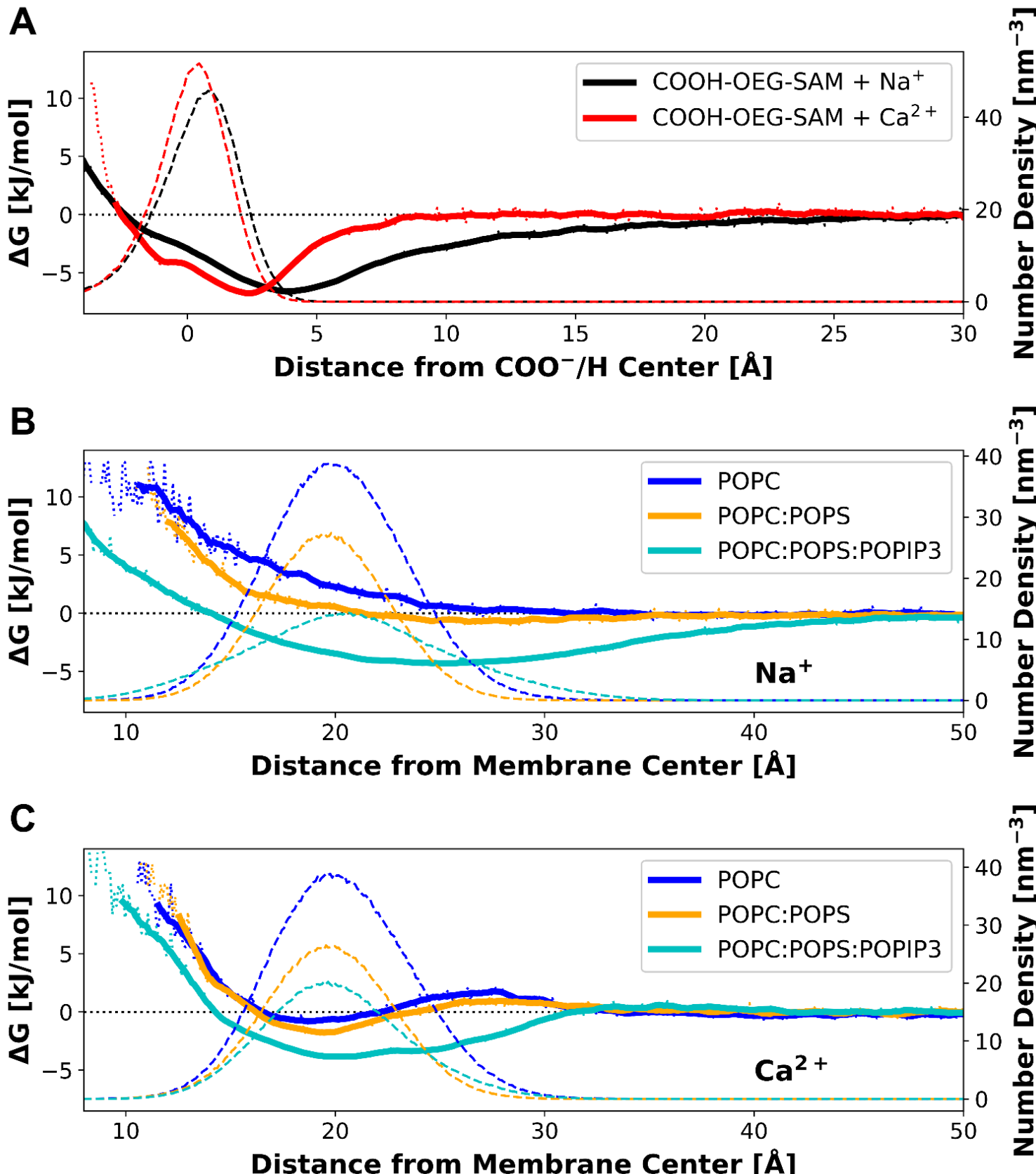

Figure 6 | Free Energy of Ion-Binding in All Atom Simulations of OEG-COOH-SAM and of Lipid Bilayer Membranes: (A) Free energy profiles for cation interactions with the OEG-COOH-SAM (solid lines). These were evaluated in the presence of $400 \mathrm{mM} \mathrm{Ca}^{2+}$ (red) and $400 \mathrm{mM} \mathrm{Na}$ (black) using the ECC2 force field. Zero on the $\mathrm{x}$-axis is equal to the centre of mass of the COOH-OEG-SAM carboxyl groups. Dashed lines represent the corresponding number density profiles of the terminal $\mathrm{COO}^{-} / \mathrm{H}$ groups of the SAM-molecules $(\mathbf{B}, \mathbf{C})$ Free energy profiles for $\mathrm{Na}^{+}(\mathrm{B})$ and $\mathrm{Ca}^{2+}(\mathrm{C})$ interactions with phospholipid bilayers (solid lines). The different colours represent the different bilayers: Blue: POPC, orange: POPC:POPS (8:2, mol:mol), cyan: POPC:POPS:POPIP3 (7:2:1, mol:mol). Zero on the $\mathrm{x}$-axis is equal to the centre of mass of the respective bilayer. Dashed lines represent the corresponding number density profiles of the lipid headgroups, including the inner phosphates. 
The minima of the free energy landscapes for $\mathrm{Na}^{+}$and for $\mathrm{Ca}^{2+}$ ions above the COOH-OEG-SAM were comparable with values of $\sim 6.7$ and $\sim 6.8 \mathrm{~kJ} / \mathrm{mol}$ (Figure 6 , A), but with closer approach for $\mathrm{Ca}^{2+}\left(\mathrm{d}_{\mathrm{MIN}}=\sim 2.3 \AA\right.$ for $\mathrm{Ca}^{2+}$ and $\sim 3.9 \AA$ for $\mathrm{Na}^{+}$). In each case there was a shoulder at -1 A suggesting a degree of penetration of cations between the OEG-carboxyl groups (as can be seen from the corresponding density profiles in Figure $\mathrm{S} 14)$. However, for $\mathrm{Ca}^{2+}$ the potential well is steeper such that cation density extends for $\sim 7 \AA$ from the carboxylates at the surface of the SAM, whereas for $\mathrm{Na}^{+}$ the potential well is broader and bulk cation concentration is not reached until > $15 \AA$ from the SAM surface. Comparable results for the standard Charmm 36 and the ECC force field parameterization are represented in the SI.

We carried out similar calculations for interactions of the cations with the various lipid bilayer surfaces (Figure 6, B/C). Two trends are seen: as was observed with the SAM, $\mathrm{Ca}^{2+}$ ions are more strongly localized at the surface than are $\mathrm{Na}^{+}$ions, corresponding to clear energy minima near the bilayer headgroups and a steeper potential well for $\mathrm{Ca}^{2+}$. Furthermore, a clear trend was observed in terms of the depth of the energy well for $\mathrm{Ca}^{2+}$ when comparing a zwitterionic POPC bilayer with a modestly anionic POPC/POPS bilayer and with a more markedly anionic POPC/POPS/POPIP3 bilayer. With increasing average surface charge density from $0 \mathrm{e} / \mathrm{nm}^{2}$ for the POPC, 0.3 $\mathrm{e} / \mathrm{nm}^{2}$ for the POPC/POPS to $1.2 \mathrm{e} / \mathrm{nm}^{2}$ for the POPC/POPS/POPIP3 bilayer, the depth of the energy well intensified for $\mathrm{Ca}^{2+}$. In agreement with this trend, the SAM showed, with a surface charge density of 1.9 $\mathrm{e} / \mathrm{nm}^{-2}$, the deepest energy well for $\mathrm{Ca}^{2+}$ compared to the various bilayers. Significant $\mathrm{Na}^{+}$binding was only observed in the presence of the highly negatively charged POPIP3. POPIP3 seemed to cause for $\mathrm{Na}^{+}$, as well as for $\mathrm{Ca}^{2+}$ an intensified breadth of the energy well, suggesting strongly fluctuating binding (see also Figure S15). So there was, in the case of the POPIP3 containing bilayer, a clear $\mathrm{Ca}^{2+}$ ion density extending more than $10 \AA$ and a clear $\mathrm{Na}^{+}$ion density extending more than $20 \AA$ from the bilayer surface were observed.

\section{Discussion}

The NR results reveal a clear trend in bilayer-to-SAM distance depending on the salt solution present between the SAM and the lipid bilayer. These trends were similar for a simple POPC and more complex and biologically relevant POPC:POPS, POPC:POPS:DOPIP3, and POPC:POPS:Chol:DGDG free floating bilayers (FFBs). All four samples yielded close approach of the lipid bilayer to the COOH-OEG-SAM surface in the presence of $2 \mathrm{mM} \mathrm{CaCl}_{2}$. In the absence of additional cations, a separation of $>200 \AA$ was observed. In the presence of $200 \mathrm{mM} \mathrm{NaCl}$ an intermediate separation distance was observed, this being greater for the anionic membranes and less for the zwitterionic POPC bilayer. These results suggest attraction between bilayer and COOH-OEG-SAM is greatest in the presence of $\mathrm{Ca}^{2+}$, with a weaker attraction in the presence of an elevated concentration of $\mathrm{Na}^{+}$.

The interplay of four main intermolecular forces is thought to control the distance between the FFBs and the COOH-OEG-SAM surface: Van der Waals (vdW) forces give rise to weak yet long range attraction between the bilayer and the surface ${ }^{[48]}$, whilst entropic Helfrich fluctuational forces ${ }^{[49]}$, as well as hydration forces can produce a repulsion between the $\mathrm{COOH}$ -

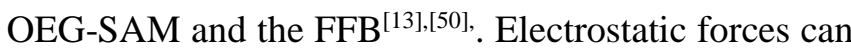
produce short range attractive or repulsive interactions depending on the surface charge of the SAM and the 
$\mathrm{FFB}^{[48],[51]}$. As both the membrane head-groups and the $\mathrm{COOH}-\mathrm{OEG}-\mathrm{SAM}$ surface contained ionisable groups and given the salt solution effects it was inferred that the predominant cause of these effects were changes in electrostatics interactions of the system under differing conditions.
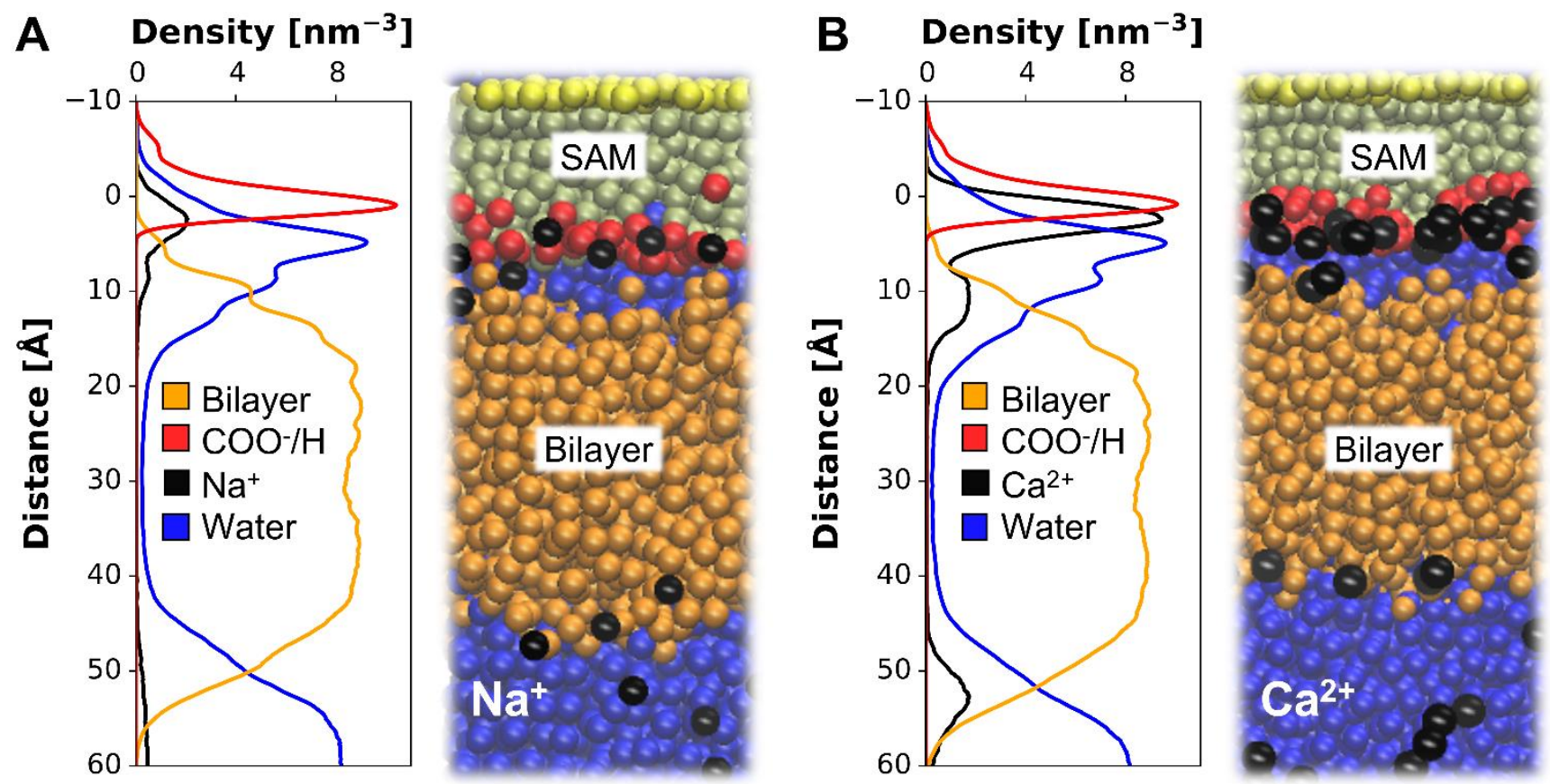

Figure 7 | Coarse Grained Free Floating Bilayer System: COOH-OEG-SAM and a POPC:POPS (8:2) bilayer were initially separated by a $12 \AA$ water layer in the presence of $400 \mathrm{mM} \mathrm{Na}{ }^{+}(\mathbf{A})$ or $\mathrm{Ca}^{2+}(\mathbf{B})$, respectively. The bilayer had a hole with a diameter of $2 \mathrm{~nm}$ throughout ions and water could pass the membrane. After $1 \mu$ s production run the number density profiles of the cations and water were determined. Zero on the y-axis is equal to the centre of mass of the COOH-OEG-SAM carboxyl groups. In order to visualize the density profiles of the ions, their density was multiplied by 10 . The $\mathrm{Ca}^{2+}$ showed a significantly stronger binding to both SAM and bilayer in comparison to $\mathrm{Na}^{+}$. The density profile of $\mathrm{Ca}^{2+}\left(\mathbf{B}\right.$, black) suggests that Ca ${ }^{2+}$ ions bridge between the anionic SAM and the phospholipid surface. However, the week binding of $\mathrm{Na}^{+}$, especially to the bilayer (A, black), might cause a too small bridging effect in order to overcome the repulsive force between the anionic SAM and an anionic bilayer. Again, the hydration layers above the SAM are observable in both cases (A/B, blue).

The MD results allow us to integrate these experimental results with an underlying molecular model of the COOH-OEG-SAM/FFB system (Figure 7). Simulations showed a layer of increased $\mathrm{Ca}^{2+}$ concentration above the SAM surface with a thickness of around $7 \AA$ and above various bilayers with a thickness of 5 to $10 \AA$ (Figure 6). Therefore, the full width of the aqueous layer between the SAM and the bilayer surfaces observed by
NR (10 to $15 \AA$ ) will contain an elevated concentration of $\mathrm{Ca}^{2+}$. Note that this would accommodate the three to four water layers seen at the SAM surface (Figure 5). A coarse-grained model of this resultant system is shown in Figure 7, B. From this integrative model it is evident that the $\mathrm{Ca}^{2+}$ ions are potentially able to bridge between the anionic SAM (carboxylate) and phospholipid surfaces. It will be of interest to examine the dynamic 
behaviour of water and $\mathrm{Ca}^{2+}$ ions within this nanoconfined salt solution layer in more detail by all atom MD simulations. In the case of $\mathrm{Na}^{+}$the much weaker binding, especially to the bilayer surfaces suggests a less intensified bridging effect of the interlayer cations, too small to overcome the repulsion force between the SAM and bilayer resulting in the intermediate position of the bilayer (Figure 7, A). Additionally all atom MD simulations comparing the behaviour of $\mathrm{Na}^{+}$with that of $\mathrm{Ca}^{2+}$ within the area between SAM and bilayer will be necessary to confirm the predicted molecular model.

\section{Conclusion}

Here we demonstrate an easily assembled floating bilayer sample system which shows an, as yet, unseen range $(\geq 200 \AA)$ of reversible bilayer movement from the surface as a result of changes in the solution cation concentrations within physiological ranges. MD simulations suggest that cations, especially $\mathrm{Ca}^{2+}$ ions electrostatically bridge the anionic membrane and SAM surfaces, while structured hydration layers above the SAM surface prevent contact between the SAM and bilayer. This "cation switchable" sample system offers a new opportunity to mimic the intracellular and extracellular environment using a planar membrane model system, which are amenable to structural and biophysical analysis, as well as sensing applications. As QCM measurements confirmed the reversible bilayer movement observed with NR, this sample system has the potential to become a standard, benchtop analysis tool investigating in vitro a myriad of membrane related biological processes such as translocation and signalling to name a few. One of the biggest advantages of our sample system is the readily fabrication. After unravelling the uncertainties about acting forces caused by ions, the manufacturing of the sample system became straightforward with being able to produce a free floating bilayer within minutes. Downstream applications of these readily fabricated sample systems could include usage in diagnostic devices containing tailored and controllable membrane models for examining the interactions of therapeutic agents, as well as potential applications in ion sensing.

Supporting information contains the following:

- Materials and Methods

- The chemical structure of the materials used in these studies.

- NR data sets and model data fits for each sample described

- QCM-D data showing FFB deposition onto a sensor surface and repeated data sets on the effect of changes in solution electrostatics.

- Mapped bond length and angle distributions of AA and CG MD for the SAM molecules

- CG MD simulation parameters for the SAM molecules

- CG SAM validation figure mentioned in main text

- ECC, ECC2, stand Charmm36 free cationbinding-energy profiles for AA SAM and AA bilayers, comparison between different force field parametrizations.

- Density profiles of cation-binding on AA SAM and AA bilayers for all force fields

- Bound ions over time for AA SAM and AA bilayers

- Number of bound cations to AA SAM and AA bilayers at equilibrium

- Table which summarizes the composition of all simulated AA SAM and AA bilayer systems 


\section{Acknowledgements}

We would like to thank Dr Arwel Hughes, Dr Martynas Gavutis, Dr Maximillian Skoda, Dr Timothy Knowles and Dr Stephen Hall for helpful advice and discussions regarding the NR and QCM-D measurements, as well as Dr Mario Campana for support on the SURF reflectometer. Furthermore, we would like to thank Dr Robin Corey, Dr Owen Vickery, Dr Irfan Alibay, Victor Everett and Michael Horrell for advice regarding the MD simulations and technical support. We would like to thank Professor Frank Heinrich and the NIST Nanofabrication facility for the metal coatings used in the NR samples described in this study. This work was supported by ISIS beam time awards 2010740 and 1920026. LHJ is funded jointly by the UKRI-BBSRC Interdisciplinary Bioscience Doctoral Training Partnership (BB/M011224/1) and the ISIS facility development studentship programme. MD Force field files, example NR data and a reprsentative model of that used in NR data fitting are availiable via doi.org/10.5281/zenodo.4434613. All NR data will be availiable via 10.5286/ISIS.E.RB2010740 from march 2023. QCM-D data is available by request.

\section{Author Contributions}

LHJ performed experiments, simulations, data analysis, and manuscript drafting. GP obtained research funding and undertook manuscript drafting. MSPS conducted research design, obtained funding, conducted data analysis and manuscript drafting. LAC conducted research design, experiments, data analysis, manuscript drafting and obtained funding

Keywords: Biological membranes, model membranes, calcium, electrostatics, neutron reflectometry, molecular dynamics, self- assembled monolayer, cation binding

[1] H. Yin, A. D. Flynn, Drugging Membrane Protein Interactions, 2016.

[2] M. Tanaka, E. Sackmann, Nature 2005, 437, 656-663.

[3] E. Sackmann, Science (80-. ). 1996, 271, 43-48.

[4] L. A. Clifton, R. A. Campbell, F. Sebastiani, J. Campos-Terán, J. F. Gonzalez-Martinez, S. Björklund, J. Sotres, M. Cárdenas, Adv. Colloid Interface Sci. 2020, 277, 102118 .

[5] C. A. Keller, K. Glasm??star, V. P. Zhdanov, B. Kasemo, Phys. Rev. Lett. 2000, 84, 5443-5446.

J. Daillant, E. Bellet-Amalric, A. Braslau, T. Charitat, G. Fragneto, F. Graner, S. Mora, F. Rieutord, B. Stidder, Proc. Natl. Acad. Sci. 2005, 102, 11639-11644. K. R. Mecke, T. Charitat, F. Graner, Langmuir 2003, 19, 2080-2087.

L. A. Clifton, N. Paracini, A. V. Hughes, J. H. Lakey, N. J. Steinke, J. F. K. Cooper, M. Gavutis, M. W. A. Skoda, Langmuir 2019, 35, $13735-13744$.

L. A. Clifton, S. A. Holt, A. V. Hughes, E. L. Daulton, W. Arunmanee, F. Heinrich, S. Khalid, D. Jefferies, T. R. Charlton, J. R. P. Webster, C. J. Kinane, J. H. Lakey, Angew. Chemie Int. Ed. 2015, 54, 11952-11955.

[16] G. Fragneto, T. Charitat, J. Daillant, Eur. Biophys. J. with Biophys. Lett. 2012, 41, 863-874.

[17] F. Lolicato, L. Joly, H. Martinez-Seara, G. Fragneto, E. Scoppola, F. Baldelli Bombelli, I. Vattulainen, J. Akola, M. Maccarini, Small 2019, DOI 10.1002/smll.201805046.

[18] I. Banerjee, R. C. Pangule, R. S. Kane, Adv. Mater. 2011, 23, 690-718.

[19] Y. He, Y. Chang, J. C. Hower, J. Zheng, S. Chen, S. Jiang, Phys. Chem. Chem. Phys. 2008, 10, 5539-5544.

[20] S. Herrwerth, W. Eck, S. Reinhardt, M. Grunze, J. Am. Chem. Soc. 2003, 125, 9359-9366.

[21] R. G. Chapman, E. Ostuni, S. Takayama, R. E. Holmlin, L. Yan, G. M. Whitesides, J. Am. Chem. Soc. 2000, 122, 8303-8304.

[22] E. Ostuni, R. G. Chapman, R. E. Holmlin, S. Takayama, G. M. Whitesides, Langmuir 2001, 17, 5605-5620.

[23] T. C. Le, M. Penna, D. A. Winkler, I. Yarovsky, Sci. Rep. 2019, 9, 1-12. 
K. P. Fears, S. E. Creager, R. A. Latour, Langmuir 2008, 24, 837-843.

G. D. Gebreeyessus, Appl. Water Sci. 2019, 9, 1-14.

P. M. Cummins, K. D. Rochfort, B. F. O'Connor, in Methods Mol. Biol. Protein Chromatogr., 2017, pp. 209-223.

L. A. Clifton, S. C. L. Hall, N. Mahmoudi, T. J. Knowles, F. Heinrich, J. H. Lakey, in Lipid-Protein Interact., Springer, 2019, pp. 96-102.

G. Van Meer, D. R. Voelker, G. W. Feigenson, Nat. Rev. Mol. Cell. Biol. 2008, 9, 112-124.

B. Fadeel, D. Xue, Crit. Rev. Biochem. Mol. Biol. 2009, 44, 264-277.

L. Boudière, M. Michaud, D. Petroutsos, F. Rébeillé, D. Falconet, O. Bastien, S. Roy, G. Finazzi, N. Rolland, J. Jouhet, M. A. Block, E. Maréchal, Biochim. Biophys. Acta - Bioenerg. 2014, 1837, 470-480.

J. Ohlrogge, J. Browse, Plant Cell 1995, 7, 957-970.

A. V Hughes, S. A. Holt, E. Daulton, A. Soliakov, T. R. Charlton, J. Steven, J. H. Lakey, S. J. Roser, J. R. Soc. Interface 2014, 11, DOI 10.1098/rsif.2014.0447.

G. Fragneto, T. Charitat, E. Bellet-Amalric, R. Cubitt, F. Graner, Langmuir 2003, 19, 7695-7702.

G. Sauerbrey, Zeitschrift für Phys. 1959, 155, 206-222.

S. B. Nielson, D. E. Otzen, Methods Mol. Biol. 2013, 974, 1-24.

S. B. Nielsen, D. E. Otzen, in Lipid-Protein Interact. Methods Protoc. (Ed.: J.H. Kleinschmidt), 2019, pp. 31-52.

S. J. Marrink, H. J. Risselada, S. Yefimov, D. P. Tieleman, A. H. De Vries, J. Phys. Chem. B 2007, 111, 7812-7824.

"Martini3 Beta," can be found under http://cgmartini.nl/index.php/martini3beta, 2020.

M. D. Porter, T. B. Bright, D. L. Allara, C. E. D. Chidsey, J. Am. Chem. Soc. 1987, 109, 3559-3568.

A. Ulman, Chem. Rev. 1996, 96, 1533-1554.

L. Strong, G. M. Whitesides, Langmuir 1988, 4, 546-558.

E. Schulze, M. Stein, J. Phys. Chem. B 2018, 122, 7699-7710.

J. Huang, A. D. MacKerell, J. Comput. Chem. 2013, 34, 2135-2145.

I. Leontyev, A. Stuchebrukhov, Phys. Chem. Chem. Phys. 2011, 13, 2613-2626.

A. Nikitin, G. Del Frate, J. Comput. Chem. 2019, 40, 2464-2472.

A. J. Pertsin, M. Grunze, Langmuir 2000, 16, 8829-8841.

J. Zheng, L. Li, S. Chen, S. Jiang, Langmuir 2004, 20, 8931-8938.

H. I. Petrache, T. Zemb, L. Belloni, V. A. Parsegian, Proc. Natl. Acad. Sci. 2006, 103, 7982-7987.

W. Helfrich, Zeitschrift fur Naturforsch. C 1973, 693-703.

A. V. Hughes, J. R. Howse, A. Dabkowska, R. A. L. . Jones, M. J. Lawrence, S. J. Roser, Langmuir 2008, 24, 1989-1999.

G. Pabst, A. Hodzic, J. Štrancar, S. Danner, M. Rappolt, P. Laggner, Biophys. J. 2007, 93, 2688-2696. 


\section{Entry for the Table of Contents}

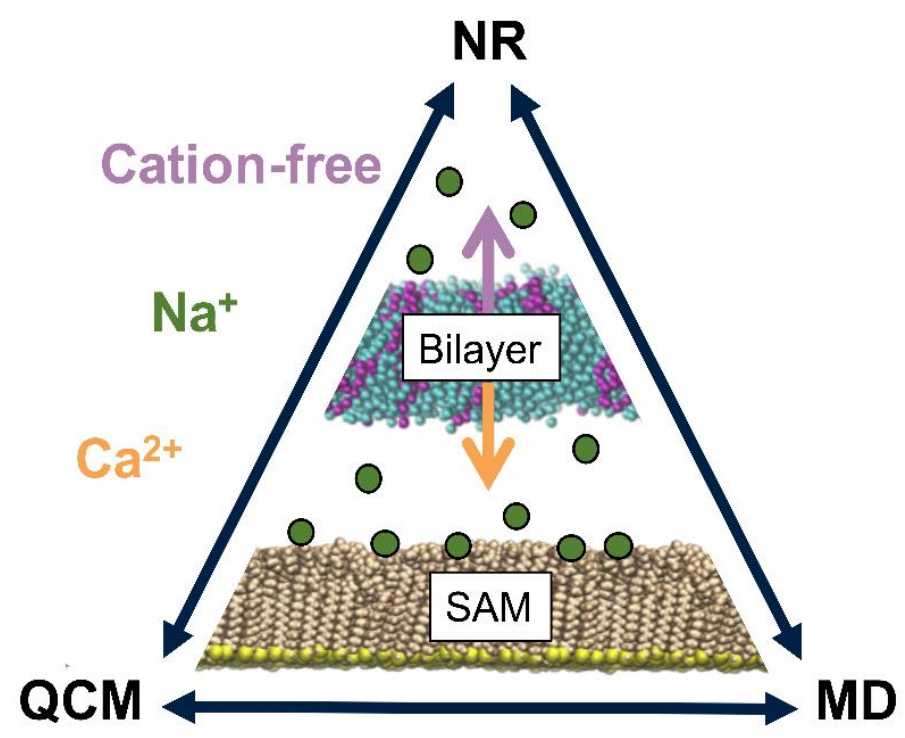

Movement of self-assembled floating bilayers up to $60 \mathrm{~nm}$ from a SAM surface was induced by $2 \mathrm{mM}$ changes in solution divalent cation concentration. A combination of neutron reflectometry, quartz crystal microbalance and molecular dynamics gave molecular level insight into the role of surface bound ions in controlling this reversible and technologically relevant effect. 